\title{
Caseating Granulomatous Inflammation: Think beyond Tuberculosis
}

Padmavathi Devi Chaganti, YVS Prabhakar, KA Seetaram, Kalyan Babu

\begin{abstract}
A 33 years old male human immunodeficiency virus (HIV) positive patient on antiretroviral therapy developed caseating granulomatous inflammation of lymph nodes. He did not respond to antituberculous treatment. Subsequently, he developed gastrointestinal tract lesions. Special stains show positivity for histoplasmosis.

The case is presented because of its resemblance to tuberculosis.
\end{abstract}

Keywords: Caseation, Granulomatous Inflammation, Tuberculosis, Histoplasmosis.

How to cite this article: Chaganti PD, Prabhakar YVS, Seetaram KA, Babu K. Caseating Granulomatous Inflammation: Think beyond Tuberculosis. Int J Phonosurg Laryngol 2013;3(2):51-54.

\section{Source of support: Nil}

Conflict of interest: None declared

\section{INTRODUCTION}

Immunocompromised state predisposes to several infections which includes both bacterial and fungal. The causes of immunocompromised state range from conditions like corticosteroid therapy to infections like human immunodeficiency virus (HIV). ${ }^{1}$ Here, we are reporting a case of histoplasmosis diagnosed as tuberculosis clinically and histopathologically in a HIV-positive patient.

\section{CASE REPORT}

A 33 years old male patient, a known case of HIV infection and on antiretroviral treatment for more than 5 years, under regular follow-up complained of fever with progressive dyspnea, productive cough with loss of appetite and weight for about 2 months.

Physical examination was unremarkable except for enlarged cervical lymph nodes and no other lymph nodes or masses were felt (Fig. 1).

Laboratory investigations showed CD4 count of 56 and total leukocyte count of 2,560 cells/cumm. X-ray of the chest showed cystic cavities hilar lymphadenopathy and pleural thickening.

Fine-needle aspiration cytology (FNAC) of cervical lymph node showed caseating granulomatous inflammation, and a diagnosis of tuberculosis was made and the patient was started on antituberculous treatment.
The patient did not respond to antituberculous treatment even after 2 months and developed difficulty in swallowing with pain in throat.

Systemic examination showed an ulcer with nodules in the soft palate (Fig. 2). A differential diagnosis of drug resistant tuberculosis, fungal infection or malignancy was considered. Biopsy was done from the edge of the ulcer in oral cavity. Microscopic examination showed hyperplasic squamous epithelium of the skin with ulceration. Subepithelium showed ill-formed granulomas consisting of foamy macrophages and epithelioid histiocytes (Figs 3 and 4). Acid-fast bacilli (AFB) staining of the lymph node lesion and oral lesion were negative.

Periodic acid schiff (PAS) stain showed yeast forms less than $5 \mu$ in macrophages confirming the diagnosis of histoplasmosis (Figs 5 and 6).

The patient was treated with amphotericin B with a dose of $1 \mathrm{mg}$ per $\mathrm{kg}$ body weight and within 2 weeks, the oral ulcers healed completely and the lymphadenopathy resolved (Fig. 7). He was started on itraconazole and remained symptom free.

\section{DISCUSSION}

Histoplasma capsulatum is a dimorphic fungus. Histoplasmosis is a systemic disease contracted by inhalation of spores of histoplasma capsulatum. ${ }^{2}$

Symptomatic disease occurs only in $10 \%$ of effected individuals. Symptomatic infection presents in three main forms that is acute pulmonary, disseminated and chronic pulmonary forms. ${ }^{3}$ In 1985 the center for disease control added disseminated form to the spectrum of conditions that characterizes the acquired immunodeficiency syndrome. ${ }^{1}$

The pathogenesis of histoplasmosis is not properly understood. The organism enters the macrophages either through opsonization or by a mechanism that appears to be specific to the fungus. ${ }^{4}$ The fungus dissociates through macrophages and spread to various organs like liver spleen, lymph nodes, bone marrow and gastrointestinal tract.

Histoplasma yeasts multiply and causes cell lysis and the products so released cause the release of gamma interferon which induces epithelioid granulomas with 


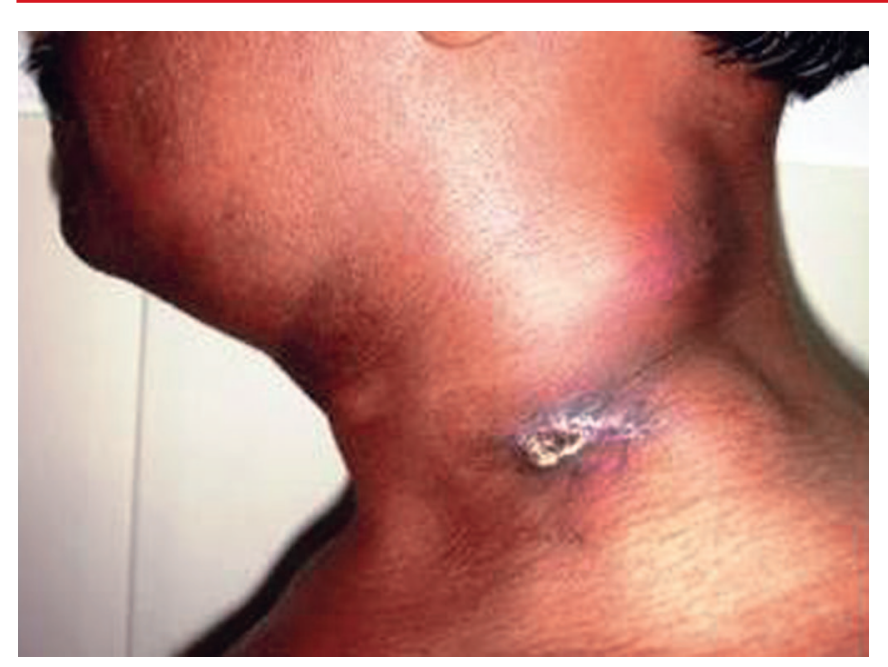

Fig. 1: Cervical lymphadenopathy

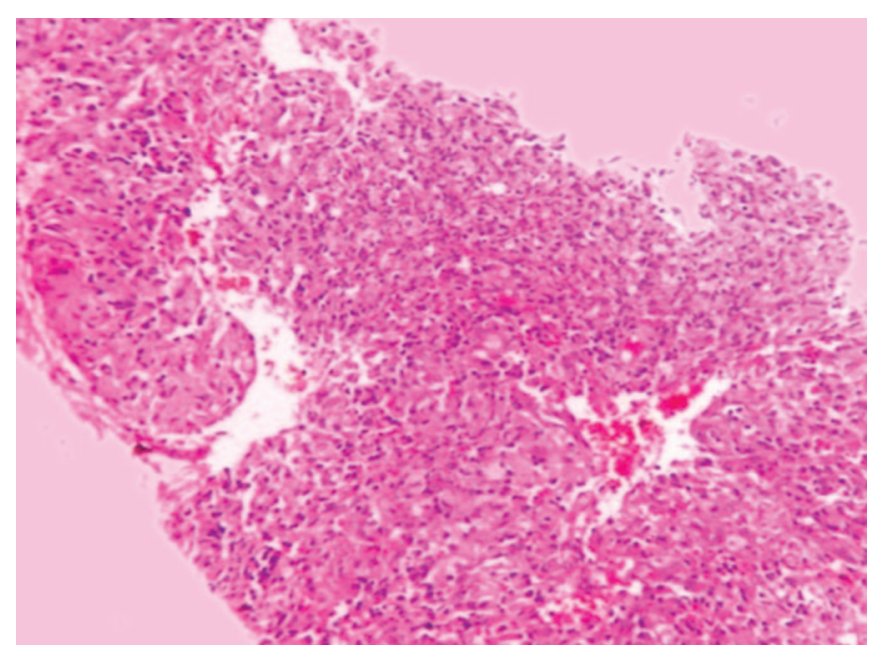

Fig. 3: Low power view of granulomas

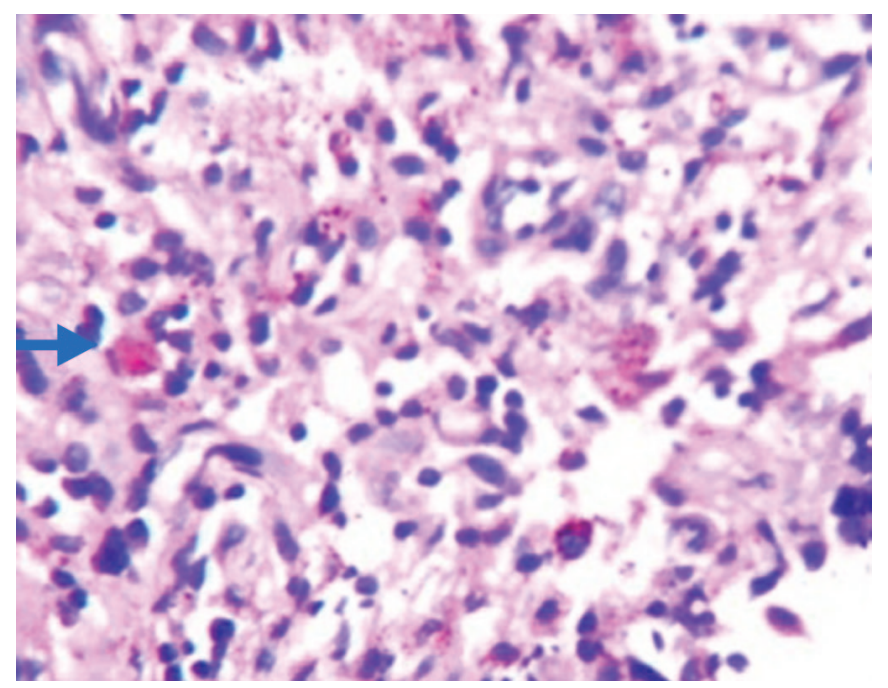

Fig. 5: PAS positivity-low power

central caseous necrosis. ${ }^{4,5}$ Histoplasma spores multiply in the lungs especially if there is previous lung injury. ${ }^{2}$

Acute pulmonary disease presents with influenza like symptoms. The initial injury is in the form of interstitial pneumonitis especially associated with centrilobular

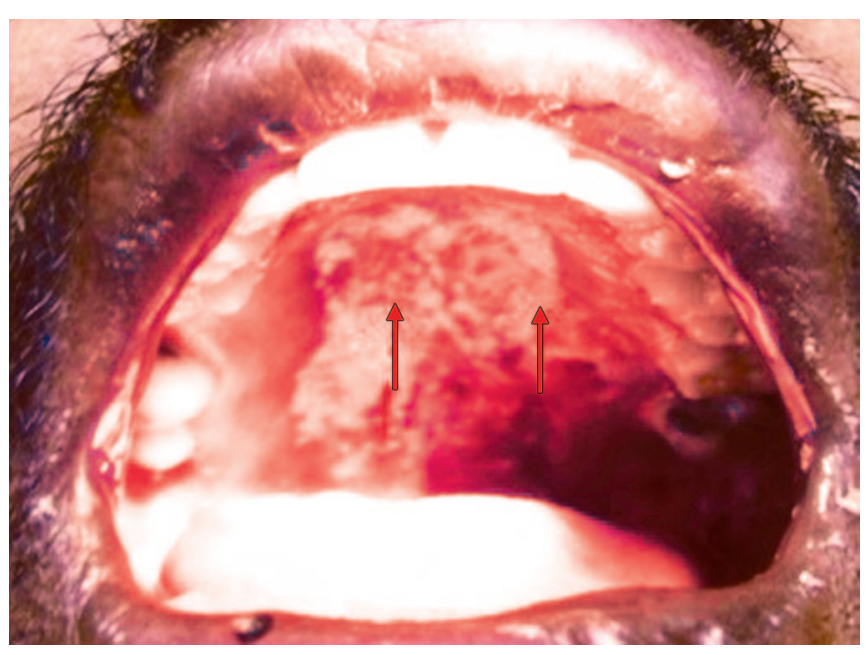

Fig. 2: Nodules on soft palate

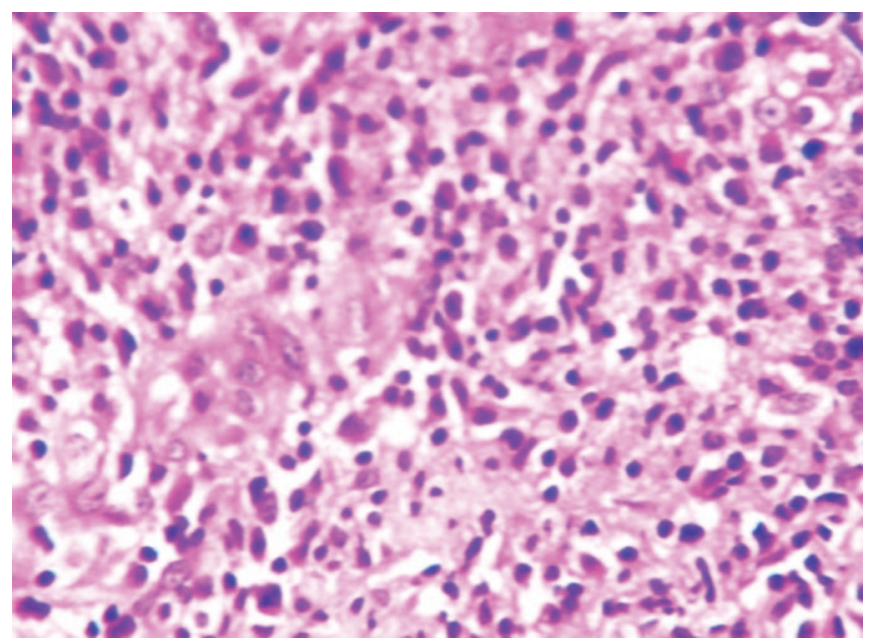

Fig. 4: High power view showing caseating granulomatous inflammation

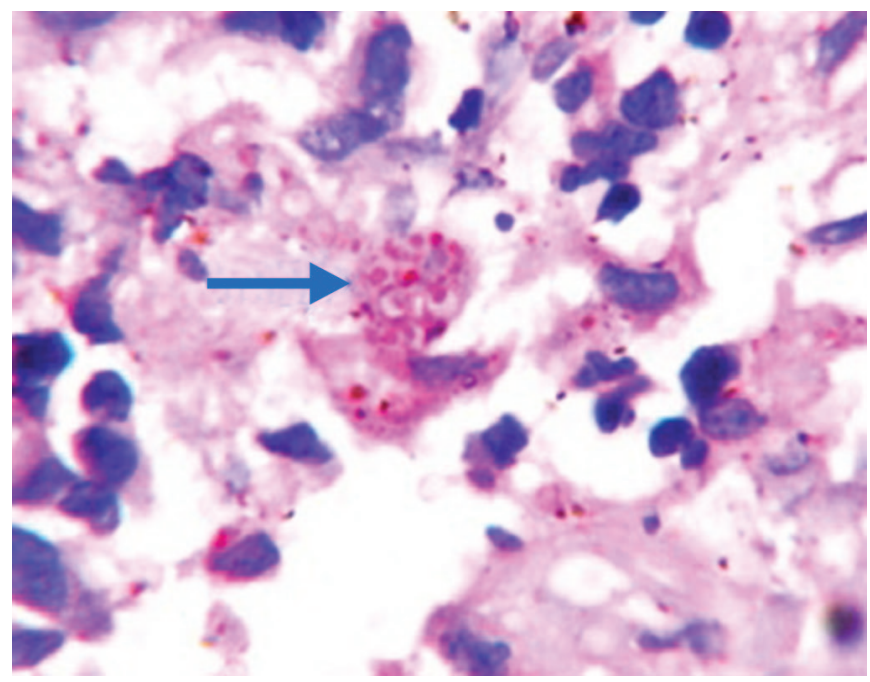

Fig. 6: PAS positivity—high power

emphysema. ${ }^{6}$ With treatment, the infection either may resolve or progress to fibrocaseous lesion.

Chronic and severe histoplasmosis is associated with pre-existing lung diseases like emphysema and lung cavities. 

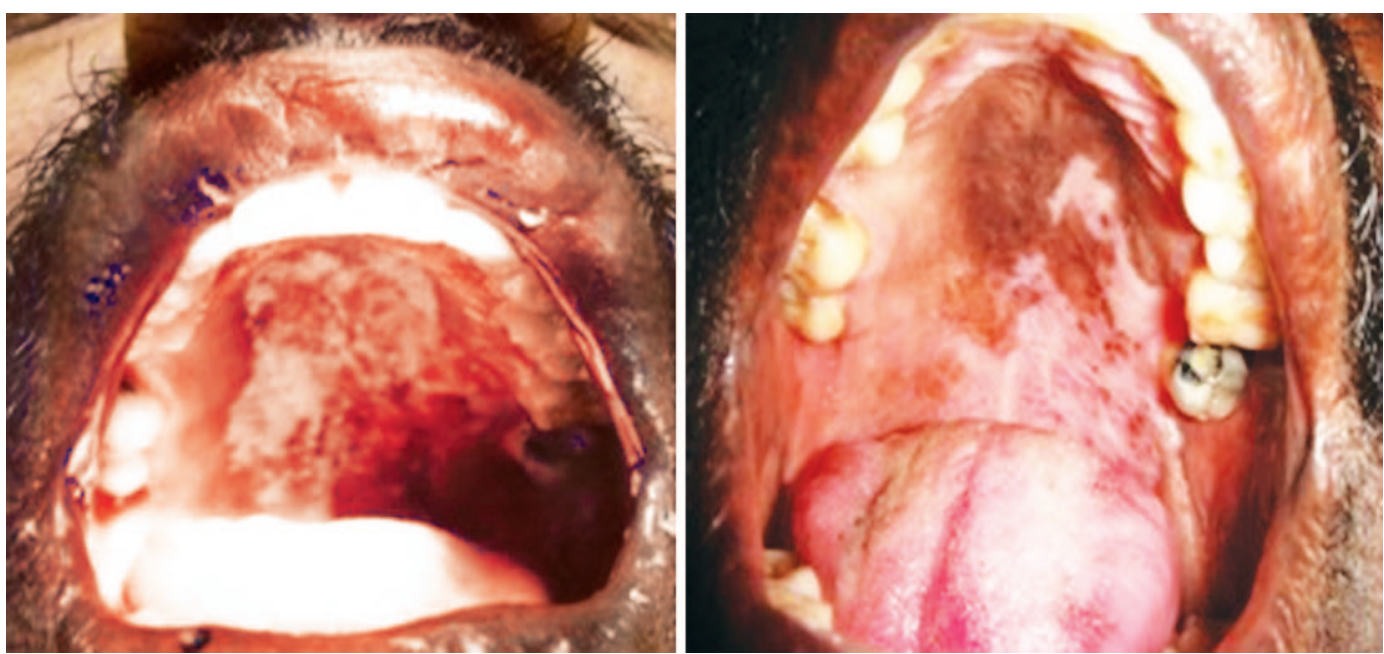

Fig. 7: Healed ulcers after treatment

Mediastinal lymphadenopathy is not observed differentiating this lesion from sarcoidosis. ${ }^{8}$

The sections from the lungs showed poorly formed epithelioid granulomas with central area of caseous necrosis. There is minimal lymphocytic response and Langhan's type of giant cells are less in number. ${ }^{8}$ Microscopic examination of the caseous material was negative for AFB. The Gomori's methenamine silver staining or PAS stain shows the typical intracellular parasites which are $4 \mu$ in size with a perinuclear halo. Plenty of intracellular and extracellular parasites are seen at the periphery of necrotic areas. ${ }^{8}$

Gastrointestinal involvement occurs because of hematogenous dissemination of the fungus. It can occur both in immunocompetent and immunosuppressed individuals. A total of 30 to $60 \%$ of patients with disseminated histoplasmosis presents with oral lesions and they are rarely primary manifestations of the disease. ${ }^{1}$

Gastrointestinal histoplasmosis presents as ulcers or nodules on the mucosa of oral cavity or the ileocecal junction or ascending colon along with lymphadenopathy. ${ }^{8}$ Microscopic findings include lymphohistiocytic infiltrations. Well-formed granulomas are rare. ${ }^{8}$

The diagnosis of fungus is confirmed by isolation of fungus from the sputum, or from bronchoscopic aspiration and staining with Grocott-Gomeri methenamine-silver staining technique. ${ }^{8}$ Confirmation is by culture on Sabouraud dextrose agar. Antibodies are detected 2 to 6 weeks after infection. ${ }^{4}$

Patients with AIDS usually present with disseminated disease. $^{5}$

Disseminated histoplasmosis present with symptoms resembling tuberculosis. ${ }^{5}$
The clinical features of disseminated histoplasmosis are fever, weight loss, fatigue, respiratory complaints, hepatomegaly splenomegaly, lymphadenopathy and bone marrow involvement. Other features include involvement of gastrointestinal tract, meninges and cardiovascular system. ${ }^{5}$

Histoplasmosis, whether presenting as pulmonary involvement, gastrointestinal or as disseminated form, both clinically and histopathologically mimics tuberculosis. ${ }^{6-8}$

Liposomal amphotericin B has proved to be more effective than conventional amphotericin because it is associated with less side effectives and better therapeutic effects. ${ }^{3}$

\section{CONCLUSION}

All cases of caseating granulomatous inflammation in HIV-positive cases have to be evaluated both by clinical methods and by special stains for both tuberculosis and histoplasmosis.

\section{REFERENCES}

1. Unis G, Servo LC. Chronic pulmonary histoplasmosis mimicking tuberculosis. J Bras Pneumol 2005;31(4):318-324.

2. Kabangila R, Semvua K, Rambabu P, Kahima J. Pulmonary histoplasmosis presenting as chronic cough, fever and massive unilateral consolidation in 15-year-old immunocompetent boy: a case report. J Med 2011;5:374.

3. Qureshi A. A case of histoplasmosis mimicking tuberculosis. J Pak Med Assoc 2008;58(8):457-458.

4. Santos JW, Michel GT, Lazzarotto M, Figaro JK, Spilmann D, Homrich GK. Chronic cavitary pulmonary histoplasmosis. J Brasileiro De Pneumologia 2009;35(11):1161-1164.

5. Chan KS, Looi LM, Chan SP. Disseminated histoplasmosis mimicking tuberculosis: a case report. Malays J Pathol 1993;15(1):155-158

6. Parikh F, Longhe VA, Khude S, Kamat R, Sandhya A. Gastrointestinal histoplasmosis mimicking abdominal tuberculosis. J Assoc Physicians India 2009;57:76-78. 
7. Mahbub S, Ahasan HN, Miah MT, Alam MB, Gupta R, Arif KM, Gupta RD, Hasnain M. Disseminated Histoplasmosis. J Med 2010;11;70-73.

8. Hernández SL, López De Blanc SA, Sambuelli RH, Roland H, Cornelli C, Lattanzi V, Carnelli MA. Oral histoplasmosis associated with HIV infection: a comparative study. J Oral Pathol Med 2004;33:445-450.

\section{ABOUT THE AUTHORS}

Padmavathi Devi Chaganti (Corresponding Author)

Professor, Department of Pathology, Guntur Medical College, Guntur Andhra Pradesh, India, Phone: 08632354749, e-mail: drcpd60@ gmail.com

\section{YVS Prabhakar}

Chief Consultant Physician, Department of Medicine, Amaravathi Institute of Medical Sciences, Guntur, Andhra Pradesh, India

\section{KA Seetaram}

Chief Consultant Dermatologist, Department of Dermatology and VD, Amaravathi Institute of Medical Sciences, Guntur, Andhra Pradesh, India

\section{Kalyan Babu}

Postgraduate, Department of Medicine, Katuri Medical College Guntur, Andhra Pradesh, India 